\title{
Cyclic heat release variability in a spark ignition engine under exhaust gas recirculation
}

\author{
Asok K. Sen ${ }^{1}$, Grzegorz $\operatorname{Litak}^{2,3, *}$, and Robert M. Wagner ${ }^{4}$ \\ ${ }^{1}$ Department of Mathematical Sciences, Indiana University, 402 North Blackford Street, Indianapolis, Indiana 46202, USA \\ ${ }^{2}$ Department of Process Control, AGH University of Science and Technology, Mickiewicza 30, PL-30-059 Krakow, Poland \\ ${ }^{3}$ Faculty of Mechanical Engineering, Lublin University of Technology, Nadbystrzycka 36, PL-20-618 Lublin, Poland \\ ${ }^{4}$ Engineering Technology Division, Oak Ridge National Laboratory, Oak Ridge, Tennessee 37831, USA
}

\begin{abstract}
We have studied cyclic heat release variability in a spark-ignition engine under exhaust gas recirculation (EGR), using nitrogen to simulate EGR. Five EGR levels are examined. We used wavelet analysis to identify the dominant modes of fluctuation and how these modes vary in time. It is found that at a low EGR level, the heat release variations exhibit high-frequency intermittent oscillations. As the EGR level increases, the high-frequency oscillations tend to become more persistent, occurring continuously over many cycles. When the EGR level is sufficiently high, intermittent oscillations are observed at both high and low frequencies. In addition, persistent low-frequency fluctuations are present at the high EGR level. We have fitted theoretical probability models to the empirical heat release distributions. Depending on the EGR level, a three-parameter probability density function such as the generalized logistic distribution, a four-parameter distribution such as Johnson SB, or the five-parameter Wakeby distribution is found to provide a good fit. The goodness of fit of the theoretical distributions is assessed by the Kolmogorov-Smirnov (KS) test statistics. A good understanding of cyclic variability is essential to develop effective control strategies for efficient combustion.
\end{abstract}

\section{Introduction}

Cycle-to-cycle variability in a combustion process is a common occurrence in spark-ignition engines. One of the possible mechanisms of this variability is the mixing of exhaust gases with fresh intake charge. Several investigators have examined the effect of recirculating exhaust gases on cyclic variations of pressure and heat release. Many of these studies are based on the methods of nonlinear dynamics [1-17]. In this paper, we examine the effect of exhaust gas recirculation (EGR) on cycle-tocycle heat release variability by using nitrogen to simulate EGR. By increasing the EGR level we covered the crossover of combustion conditions. Starting from a stable combustion process we examined lean intermittent combustion and finally the absence of combustion in the high limit of EGR. Physically, the cycle-to-cycle fluctuations appear and evaluate directly due to the changes in EGR which is was identified by Heywood [1]. This idea was later considered in several papers $[9,11$ $13,16]$ to build the simple combustion model based on each cycle amounts of air, fuel and residual gases in combustion chamber.

In this paper, we examine the effect of exhaust gas recirculation (EGR) on cycle-to-cycle heat release variability by using nitrogen to simulate EGR. Five EGR levels are examined. We use wavelet analysis to identify the dominant modes of fluctuation and how these modes vary in time. It is found that at a low EGR level, the heat release variations exhibit high-frequency intermittent oscillations. As the EGR level increases, the highfrequency oscillations tend to become more persistent, occurring continuously over many cycles. When the EGR level is sufficiently high, intermittent oscillations are observed at both high and low frequencies. In addition, persistent low-frequency fluctuations are present at the high EGR level. We have fitted theoretical probability models to the empirical heat release distributions. Depending on the EGR level, a three-parameter probability density function such as the generalized logistic distribution, a four-parameter distribution such as Johnson SB, or the five-parameter Wakeby distribution is found to provide a good fit. The goodness of fit of the theoretical distributions is assessed by the KolmogorovSmirnov (KS) test statistics [18]. A good understanding of cyclic variability is essential to develop effective control strategies for efficient combustion.

Our presentation is organized as follows. First, we briefly describe the experimental procedure. This is followed by a review of wavelet analysis methodology and its application to the heat release data. Next, we discuss the theoretical probability models that are used to fit the data. Finally, a few concluding remarks are given.

\section{Experimental setup and procedure}

The experimental set up used in the present investigation is depicted in Figure 1. Experiments were performed in

${ }^{*}$ Corresponding author: g.litak@pollub.pl 
the Internal Combustion Engines Laboratory at the Missouri University of Science and technology, Rolla, USA. The research engine is a Ricardo Hydra singlecylinder engine, No. 107. The engine was built specifically by Ricardo Consulting Engineers to match the bore, stroke, and head bolt pattern of a Ford Zetec 2.0 Liter 4-cyclinder gasoline engine. The engine uses the piston, piston rings and connecting rods from a Zetec engine. The Zetec geometry produces charge motion and enhanced mixing in the cylinder representative of modern SI engines.

The engine was coupled to a $22 \mathrm{~kW}$ (30 HP) Baldor Vector Drive dynamometer. The dynamometer can motor a non-fired engine or absorbing a load from a firing engine. The dynamometer switches between absorbing and applying power in order to maintain a constant preset speed. The ability of the dynamometer to maintain a constant speed is essential for performing experiments at high EGR levels, since under these conditions the engine behavior may be very erratic and partial fires or misfires may occur. The in-cylinder pressure was measured with a Kistler model 6010B piezoelectric pressure transducer. The output of the pressure transducer was passed through a Kistler model 5010 charge amplifier to produce a voltage proportional to the pressure. The voltage was digitized and stored on a computer using a data acquisition board (see [19] for details). Measurements were made for approximately 1000 consecutive cycles of engine operation. From the pressure measurements, the amount of heat release was calculated using a procedure based on the first law of thermodynamics [1].

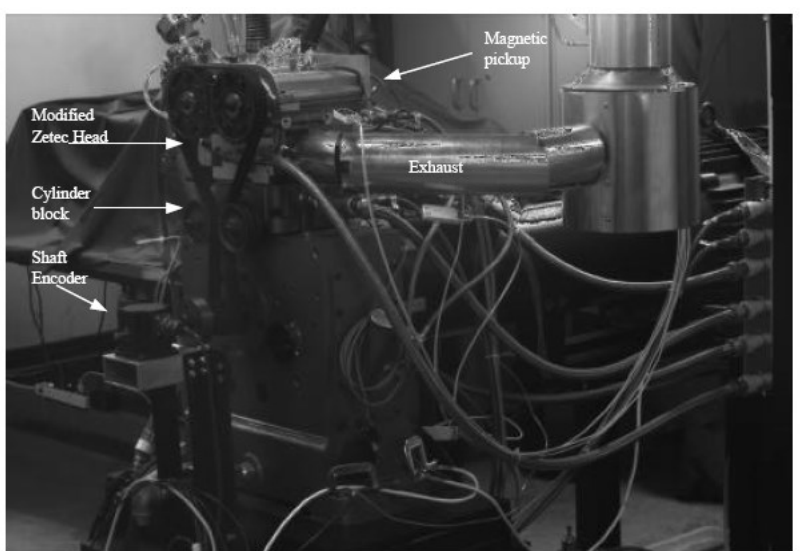

Fig. 1. Experimental set up showing the Ricardo Hydra research engine.

The effects of EGR on CO and NOx emissions, and fuel efficiency have been investigated by several researchers [20-22], while the cycle-to-cycle combustion fluctuations in [23-31].

In our experiments, the EGR level was varied while maintaining a constant fuel-oxidizer ratio at the stoichiometric value. The amount of nitrogen was metered using a Teledyne-Hastings HFM-60 thermal flow meter. These experiments are particularly relevant for reduction of CO and NOx emissions in automobiles. Since the threeway catalysts used to reduce the harmful emissions require a stoichiometric mixture to operate properly [1], experiments on varying EGR with a constant stoichiometric ratio can be used effectively.

\section{Wavelet Analysis}

A wavelet is a small wave with zero mean and finite energy. Consider a time series $\left\{x_{i}\right\}$ with $i=1,2,3, \ldots, N$. The continuous wavelet transform (CWT) of this time series with respect to a wavelet $\psi(t)$ is given by the convolution of the time series with a scaled and translated version of $\psi(t)$. The function $\psi(t)$ is referred to as an analyzing wavelet or a mother wavelet. The convolution is expressed by [32-34]

$$
W_{n}(s)=\sum_{n^{\prime}=1}^{N}\left(\frac{\delta t}{s}\right)^{1 / 2} x_{n^{\prime}} \psi^{*}\left[\frac{\left(n^{\prime}-n\right) \delta t}{s}\right] .
$$

Here $\delta t$ is the sampling interval, and an asterisk on $\psi$ denotes its complex conjugate. The symbols $s$ and $n$ (or $\left.n^{\prime}\right)$ are called scale and time index, respectively. The scale parameter controls the dilation $(s>1)$ and contraction $(s<$ 1 ) of the mother wavelet. The time index, $n$, indicates the location of the wavelet in time; in other words, as $n$ varies, the signal is analyzed in the vicinity of this point. The amount of signal energy contained at a specific scale $s$ and location $n$ is given by the squared modulus of the CWT and is referred to as the wavelet power spectrum $\left|W_{n}(s)\right|^{2}$

. The wavelet power spectrum (WPS) is a measure of the variance at different scales or frequencies. The WPS which depends on both scale and time is represented by a surface. By taking contours of this surface and plotting them on a plane, a time-scale representation of the spectrum may be derived. From the time-scale representation of the WPS, the dominant spectral modes of variability and the time interval over which these modes may persist can be discerned by visual inspection. A time-scale representation is found to be useful for extracting important features of signals arising in many applications. An alternate representation, namely, a timefrequency representation has also been used. A scale-tofrequency conversion, which follows a reciprocal relationship, can be easily made by use of the formula: $f=f_{0} f_{*} / s$, where $f$ is the instantaneous frequency of the signal, $f_{*}$ is the sampling frequency, and $f_{0}$ is the center frequency of the mother wavelet (see below). In our analysis we used a complex Morlet wavelet as the mother wavelet. A complex Morlet wavelet consists of a plane wave modulated by a Gaussian function and is described by:

$$
\psi(\eta)=\pi^{-1 / 4} e^{i \omega_{0} \eta} e^{-\eta^{2} / 2}
$$

Here $\omega_{0}=2 \pi f_{0}$ is referred to as the order of the wavelet, with $f_{0}$ being the center frequency. The value of $\omega_{0}$ controls the number of oscillations that is present in the mother wavelet and thus influences the frequency and time resolutions of the corresponding wavelet transform.

A larger value of $\omega_{0}$ provides a higher frequency resolution whereas a smaller value improves the time resolution. A Morlet wavelet of order 6 is often chosen as the mother wavelet. This choice provides a good balance between time and frequency localizations. For this choice, 
the scale is approximately equal to the Fourier period and thus the terms scale and period can be used interchangeably for interpreting the results. Wavelet analysis has been used in a wide variety of applications. In our recent work, we have employed continuous wavelet transform to study cyclic variability in the combustion process of various engines [23,27,34-35].

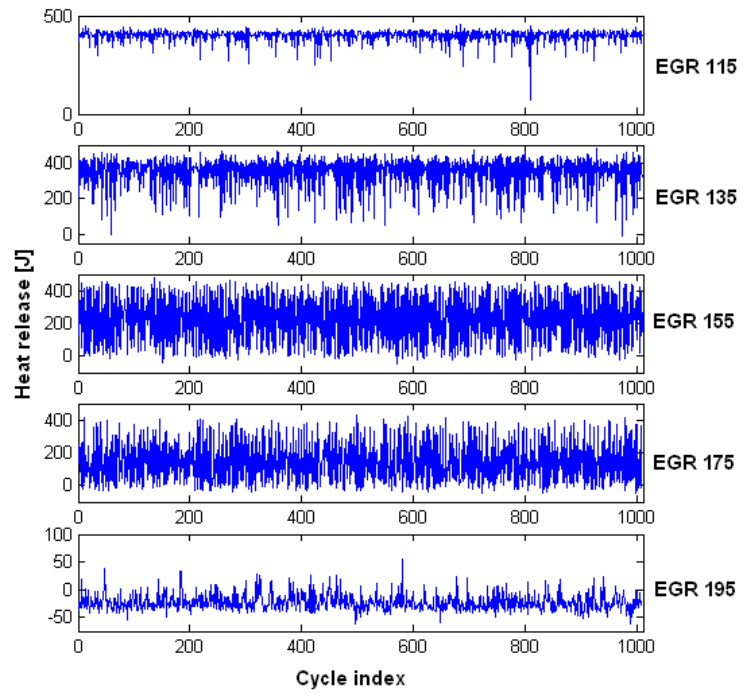

Fig. 2. Time series of heat release variations for EGR levels of (a) $17.22 \%$, (b) $20.26 \%$, (c) $23.12 \%$, (d) $26.15 \%$, and (e) $29.05 \%$.

We now discuss the results of our wavelet analysis of the heat release data for different EGR levels. The time series of the heat release variations for percent EGR levels of $17.22,20.26,23.12,26.15$, and $29.05 \%$ are plotted in Fig. 2. The WPS of these time series are displayed in Fig. 3. Consider first the WPS shown in Figure 3 a for $17.22 \%$ EGR level. It is apparent from this figure that at this level of EGR, the heat release fluctuations exhibit a highfrequency intermittent pattern in the 2-8 cycle band. In addition, intermittent oscillations are observed around the 12-cycle band.

(a) EGR 115

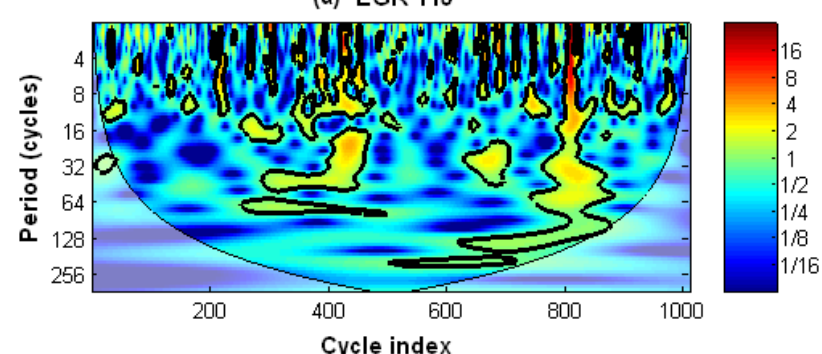

Cycle index
(b) EGR 135

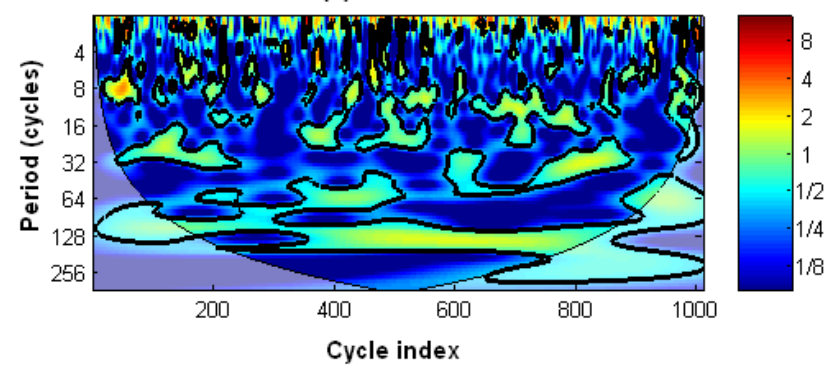

(c) EGR 155

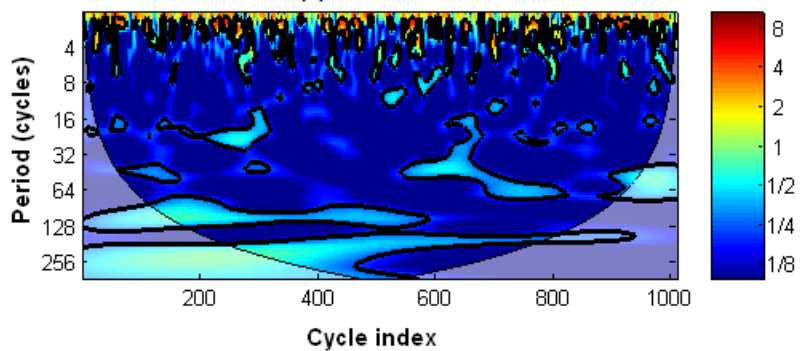

(d) EGR 175

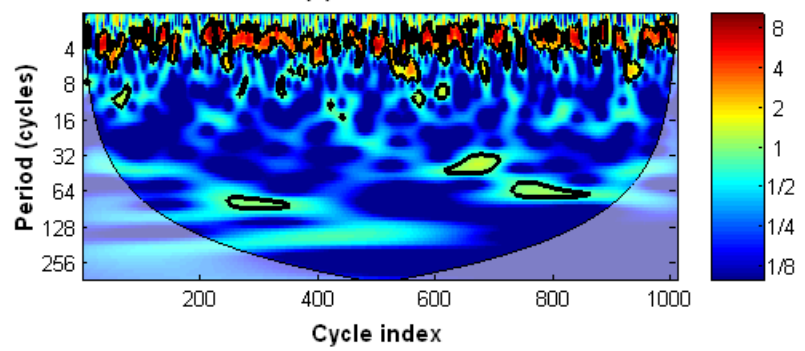

(e) EGR 195

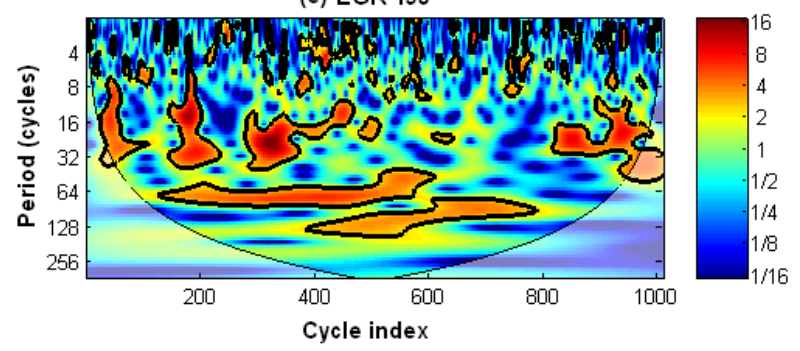

Fig. 3. Wavelet power spectrum (WPS) of each of the heat release time series shown in Fig. 2. EGR levels are: (a) $17.22 \%$, (b) $20.26 \%$, (c) $23.12 \%$, (d) $26.15 \%$, and (e) $29.05 \%$.

Next, we consider the EGR level of $20.26 \%$ for which Figure $3 \mathrm{~b}$ applies. At this EGR level, the high-frequency (2-8 cycle) intermittent patterns exist together with several weaker low-frequency oscillations that persist over several engine cycles. As the EGR level is increased to $23.12 \%$ and $26.15 \%$, the high-frequency oscillations become more persistent spanning many cycles, and the low-frequency fluctuations tend to disappear (Figures 3c, d). Finally, when the EGR level is increased to $29.05 \%$, Figure $3 \mathrm{e}$ reveals that intermittent oscillations appear in the 2-8 cycle band. Intermittent fluctuations are also observed around the low frequency 24-cycle band. Furthermore, we see more persistent low-frequency variations around the 64-cycle band spanning approximately 125 to 575 cycles.

\section{Heat Release Probability Distributions}

The statistical properties of the heat release data for the different EGR levels considered here are listed in Table 1. The histograms of the data are depicted in Fig. 4. Clearly the histograms show that the heat release variations deviate from a normal or Gaussian distribution. This can also be inferred from the skewness and kurtosis values presented in Table 1. Note that for a normal distribution, the skewness is zero, and the kurtosis has the value 3 . 
(a) EGR 115

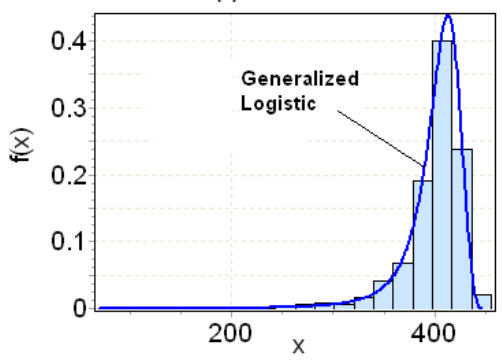

(b) EGR 135

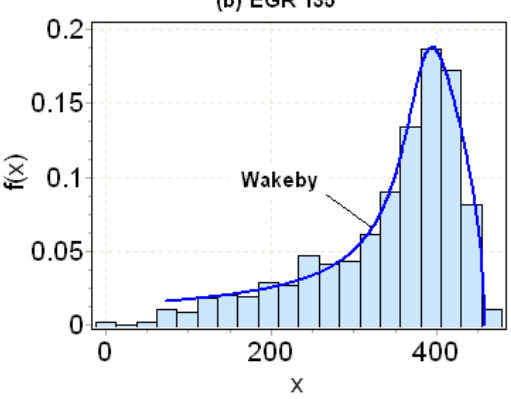

(c) EGR 155

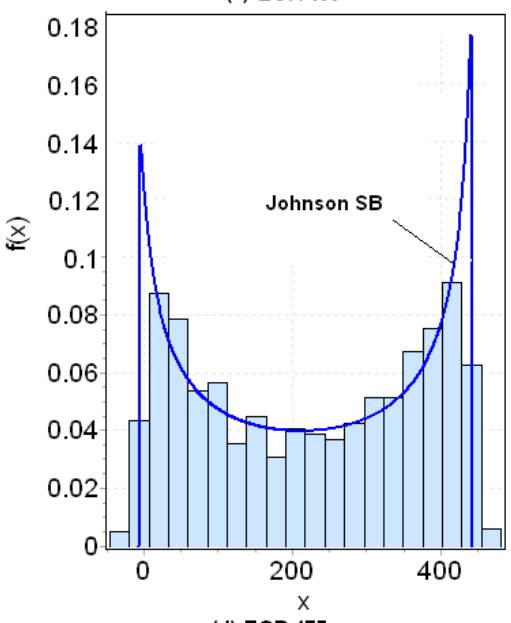

(d) EGR 175

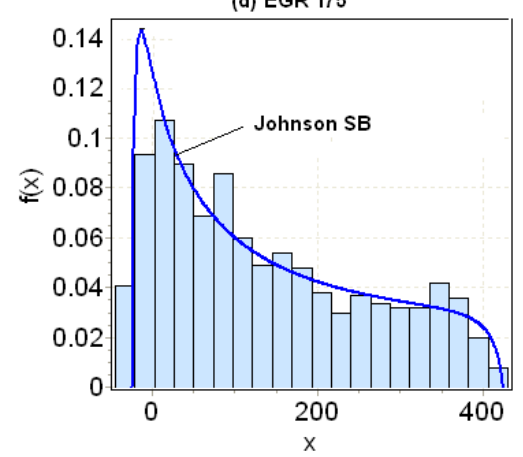

(e) EGR 195

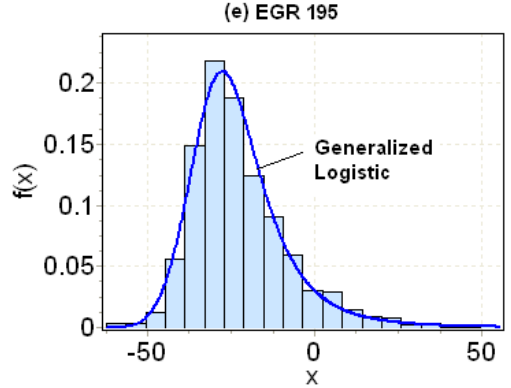

Fig. 4. Corresponding histograms of each of the heat release time series shown in Fig. 2. EGR levels are: (a) $17.22 \%$, (b) $20.26 \%$, (c) $23.12 \%$, (d) $26.15 \%$, and (e) $29.05 \%$.
Table 1. Statistics of the time series (see Fig. 2): EGR, Mean, standard deviation DS, skewness, and kurtosis.

\begin{tabular}{|l|l|l|l|l|}
\hline EGR & $\begin{array}{l}\text { Mean } \\
{[\mathrm{J}]}\end{array}$ & SD [J] & Skewness & Kurtosis \\
\hline $\begin{array}{l}115 \\
(17.22 \%,)\end{array}$ & 398.6 & 29.8 & -2.98 & 21.6 \\
\hline $135(20.26 \%)$ & 343.0 & 89.5 & -1.23 & 3.94 \\
\hline $155(23.12 \%)$ & 225.3 & 151 & -0.06 & 1.54 \\
\hline $175(26.15 \%)$ & 135.9 & 125 & 0.58 & 2.12 \\
\hline $195(29.05 \%)$ & -22.7 & 14.4 & 1.12 & 5.16 \\
\hline
\end{tabular}

We have fitted several theoretical (non-Gaussian) distributions to these data. Among them, depending on the EGR level, a three-parameter generalized logistic distribution, a four-parameter Johnson SB distribution, or a five-parameter Wakeby distribution is found to provide a good fit. Note that the intermittency is signaled by larger discrepancy from the Gaussian value. Namely for EGR 115 and 195, which is consistent with the wavelet analysis (see Figs. 3a and e, respectively).

Increasing EGR level we evolve from good to poor combustion characterized by the single peak probability distributions with different inclinations. Note the skewers is changing the sign (Tab. 1). In the middle of the considered EGR levels the combustion process is characterized by double peak probability pressure distribution corresponding to different combustion in these two opposite limits of good and poor combustion.

The theoretical probability density functions are superimposed on the corresponding histograms in Fig. 4. A brief description of the theoretical distributions is given in Appendix A. The goodness of fit of the theoretical distributions was estimated on the basis of the Kolmogorov-Smirnov test statistics. For each distribution, the null hypothesis that the theoretical distribution provides a good fit was found to hold at a significance level of 0.05 or less.

\section{Concluding Remarks}

We have examined the cycle-to-cycle heat release variations in a spark ignition engine at different levels of EGR, using nitrogen to simulate EGR. The EGR levels were varied by maintaining the fuel-oxidizer ratio at the stoichiometric value. Our results indicate that at low EGR levels, the heat release variations exhibit high-frequency intermittent oscillations together with weaker, persistent low-frequency oscillations. As the EGR level increases, the high-frequency oscillations become persistent while the low-frequency fluctuations tend to disappear. When the EGR is sufficiently high, intermittent oscillatory patterns are observed at both high and low frequencies. In addition, persistent low-frequency fluctuations occur at the high EGR level. These results may be useful to develop effective control strategies for efficient combustion in spark ignition engines [14-19].

Finally, it is worth noting that the observed intermittency leads to the specific bifurcation scenario (see the sequence Figs. 2a-e). Following generalized logistic dynamics, in the larger and small EGR levels, one used to observe the 3-cycle period which is unstable to transition into chaotic oscillations. Thus, the chaotic oscillations could be responsible for the EGR cases of 
20.26 and 26.15. Indeed, on the wavelet power spectra we identify fluctuations with multiple periods (Fig. 3b and d). The problem appearing the intermittency was also illustrated in terms histograms, and expressed in statistical description by using standard deviation, skewness and kurtosis.

However, the actual situations are more complicated because of the influence additional random fluctuations. The sources of these fluctuations are both external and internal [1], such as fluctuating mixture composition due to imperfections in injection procedure and the spatial non-uniformity of mixture. Similar intermittent transitions were observed in the recent works on HCCI (homogenous charge compression ignition) engines where the EGR plays the important role [27,36-37].

\section{References}

1. J.B. Heywood, Internal Combustion Engine Fundamentals (McGraw -Hill, New York 1988).

2. D.J. Patterson, SAE paper No. 660129 (1966).

3. R.E. Winsor and D.J. Patterson, SAE paper No. 730086 (1973).

4. J.C. Kantor" Science 224, 1233 (1984).

5. J.W. Daily, Combustion Science and Technology $\mathbf{5 7}$, 149 (1988).

6. A.P. Foakes and D.G. Pollard, Combustion Science and Technology 90, 281 (1993).

7. L. Chew, R. Hoekstra, J.F. Nayfeh, and J. Navedo, SAE Paper No. 942486 (1994).

8. Z. Hu, SAE paper No. 961197 (1996).

9. C.S. Daw, C.E.A. Finney, J.B. Green Jr., M.B. Kennel, J.F. Thomas, and F.T. Connolly, SAE paper No. 962086 (1996).

10. C. Letellier, S. Meunier-Guttin-Cluzel, G. Gouesbet, F. Neveau, T. Duverger, and B. Cousyn SAE Paper No. 971640 (1997).

11. C.S. Daw, M.B. Kennel, C.E.A. Finney, and F.T. Connolly, Physical Review E 57, 2811 (1998).

12. R.M. Wagner, J.A. Drallmeier, and C.S. Daw, International Journal of Engine Research 1, 301 (2001).

13. C.S. Daw, C.E.A. Finney, and E.R. Tracy, Review of Scientific Instruments 74, 915-930 (2003).

14. M. Wendeker, G. Litak, J. Czarnigowski, K. Szabelski, International Journal of Bifurcation and Chaos 14, 1801 (2004).

15. T. Kamiński, M. Wendeker, K. Urbanowicz, G. Litak, Chaos 14, 401 (2004).

16. P.L. Curto-Risso, A. Medina, A. Calvo Hernández, L. Guzmán-Vargas, F. Angulo-Brown, Applied Energy 88, 1557 (2011).

17. P.L. Curto-Risso, A. Medina, A. Calvo Hernández, L. Guzmán-Vargas, F. Angulo-Brown, Physica A 389 5662 (2010).

18. P.A.W. Lewis, E.J. Orav, Simulation Methodology for Statisticians, Operations Analysts, and Engineers, CRC Press, 1988.

19. R.W. Sutton, Investigation of cyclic dispersion under lean fueling and high levels of simulated EGR, Master's thesis, University of Missouri, Rolla, 2000.
20. C. De Petris, S. Diana, V. Giglio, G. Police, SAE Paper No. 941933 (1994).

21. H.E. Jääskeläinen, J.S. Wallace, SAE Paper No. 942006 (1994).

22. M. Tabata, T. Yamamoto, T. Fukube, SAE Paper No. 950684 (1995).

23. A.K. Sen, G. Litak, R. Taccani, R. Radu, Chaos, Solitons \& Fractals, 38, 886 (2008).

24. G. Lumsden, G. Eddleston, R. Sykes, SAE Paper No. 970505 (1997).

25. M.B. Young, SAE Paper No. 810020 (1981).

26. C.S. Daw, K.D Edwards, R.M. Wagner, and J.B. Green, Jr, Journal of Engineering for Gas Turbines and Power 130, 052801 (2008).

27. A.K. Sen, G. Litak, K.D. Edwards, C.E.A. Finney, C.S. Daw, R.M. Wagner, Appl. Energy 88, 1557 (2011).

28. J. Hunicz, M. Geca, A. Rysak, G. Litak, P. Kordos, J. Vibroengineer. 15, 1093 (2013).

29. M. Geca, G. Litak, Measurement 108, 18 (2017).

30. S.L. Ding, E.Z. Song, L.P. Yang, G. Litak, C. Yao, X.Z. Ma, Chaos, Soliliton \& Fractals 93, 99 (2016).

31. S.L. Ding, E.Z. Song, G. Litak, L.P. Yang, Y.Y. Wang, X.Z. Ma, Appl. Therm. Engineer. 121, 768 (2017).

32. C. Torrence, G.P. Compo, Bulletin of the American Meteorological Society 79, 61 (1998).

33.P. Kumar, E. Foufoula-Georgiou, Reviews in Geophysics 35, 385 (1997).

34. A.K. Sen, G. Litak, C.E.A. Finney, C.S. Daw, R.M. Wagner. Appl. Energy 87, 1736 (2010).

35. M. Geca, M. Wendeker, G. Litak, J. Vibroengineer., 14, . 582 (2012)

36. K. Matsumoto, I. Tsuda, and Y. Hosoi, Zeitschrift für Naturforschung 62, 587 (2007).

37. B.C. Kaul, J.B. Vance, J.A. Drallmeier, J. Sarangapani, Proceedings of the Institution of Mechanical Engineers Part D: Journal of Automobile Engineering 223, 423 (2009).

38. G.D. Gettinby, C.D. Sinclair, D.M. Power, R.A Brown, Journal of Business, Finance and Accounting 31, 607 (2004).

39. N.L. Johnson, Biometrika 36, 149 (1949).

40. K.O. Bowman, L.R. Shenton, Encyclopedia of Statistical Sciences 4, 303 (1983).

41.S. Kotz, J.R. van Dorp, Beyond beta: Other continuous families of distributions with bounded support and applications. (World Scientific, Singapore 2004).

42. L.W. Swift, H.T. Schreuder, Mon. Weather Rev. 109, 2535 (1981).

43. E.A., Pani, D.R. Haragan, Water Resour. Bull. 21, 393-405 (1985).

44. M.R., Flynn, Ann. Occup. Hyg. 48, 617 (2004).

45. K. Rennolls, M. Wang, Can. J. Forest Res. 35, 575 (2005).

46. J.R.M. Hosking, The Wakeby distribution. Research Report RC12302, (IBM Research Division, Yorktown Heights, New York 1986).

47. J.S., Park, H.S. Jun, R.S. Kim, J.H. Oh, Int. J. Climatol. 21, 1371 (2001). 


\section{Appendix A. Theoretical Probability Models}

\section{(a) Generalized Logistic Distribution}

The generalized logistic distribution is an extension of the well-known logistic distribution and is used in many applications [38]. It is defined by the following probability density function:

$$
f(x)= \begin{cases}\frac{(1-k z)^{-1-1 / k}}{\sigma\left(1+(1+k z)^{-1 / k}\right)^{2}} & k \neq 0 \\ \frac{\exp (-z)}{\sigma(1+\exp (-z))^{2}} & k=0\end{cases}
$$

with

$$
\begin{aligned}
& 1+k \frac{(x-\mu)}{\sigma\left(1+(1+k z)^{-1 / k}\right)^{2}}>0 \text { for } k \neq 0 \\
& -\infty<x<+\infty \quad \text { for } k=0 .
\end{aligned}
$$

Here

$z \equiv \frac{x-\mu}{\sigma}$

is the standardized variable, and the symbols $k, \sigma$, and $\mu$ denote the shape, scale and location parameters, respectively. The corresponding cumulative distribution functions are:

$$
F(x)=\left\{\begin{array}{ll}
\frac{1}{1+(1+k z)^{-1 / k}} & k \neq 0 \\
\frac{\exp (-z)}{1+\exp (-z)} & k=0
\end{array} .\right.
$$

\section{(b) Johnson SB Distribution}

The Johnson SB distribution belongs to a family of distributions introduced by Johnson [39]. The letter SB denotes that it is a bounded distribution. Various aspects of these distributions have been developed by Bowman and Shenton [40] and Kotz and van Dorp [41], among others. The Johnson SB distribution, which is also referred to as the 4-parameter lognormal distribution, is described by the cumulative distribution function (cdf):

$$
F(x)=\Phi\left(\gamma+\delta \ln \left[\frac{x-\xi}{\lambda+\xi-x}\right]\right),
$$

with $\xi \leq x \leq \xi+\lambda$. Here $\lambda$ and $\xi$ are the scale and location parameters, respectively; $\gamma$ and $\delta$ are the shape parameters, and the function $\Phi$ denotes the Laplace integral (see [40] for details):

The Johnson SB distribution has found applications in numerous situations including modeling of precipitation events $[42,43]$, toxicological studies involving human exposure to airborne pollutants [44], and forest ecology and management [45].

\section{(c) Wakeby Distribution}

Let $X$ be a random variable, and $F(x)=P(X \leq x)$ where $P$ defines the probability and $F(x)$ is the corresponding cdf. The Wakeby distribution is defined as a distribution whose inverse cdf, $x(F)$, is given by

$$
x(F)=\xi+\frac{\alpha}{\beta}\left[1-(1-F)^{\beta}\right]-\frac{\gamma}{\delta}\left[1-(1-F)^{-\delta}\right] .
$$

Here the range of $x$ is:

$$
\begin{gathered}
\xi \leq x<\infty \text { if } \delta \geq 0 \text { and } \gamma>0 \\
\xi \leq x \leq \xi+\alpha / \beta-\gamma / \delta \text { if } \delta<0 \text { or } \gamma=0 .
\end{gathered}
$$

The symbols $\alpha, \beta, \gamma$, and $\delta$ are called shape parameters, and $\xi$ is referred to as the location parameter [46].

In order that the function $x(F)$ given by Eq. (A4) represents an inverse cumulative density function, certain restrictive conditions should be imposed among the various parameters (see [46] for details).

By the Wakeby distribution contains five parameters, it can take a wider range of shapes than those with fewer parameters. Accordingly, it offers a lot more flexibility for fitting different types of data than other distributions. For specific choices of the various parameters, the Wakeby distribution mimics the shapes of many skewed distributions (such as lognormal, generalized extreme value, and generalized Pareto) that are often used. Note, however, that in contrast to the generalized logistic and Johnson SB distributions, the cdf or pdf of the Wakeby distribution cannot be expressed in closed form.

The cumulative distribution function, $F(x)$, is computed by numerically inverting the function given in Eq. (A4). The probability density function, $f(x)$, which is the derivative of $F(x)$, is subsequently found from the relation [39]:

$$
f(x)=\frac{[1-F(x)]^{\delta+1}}{\gamma+\alpha[1-F(x)]^{\beta+\alpha}} .
$$

Since its introduction, the Wakeby distribution has been used in several applications (see, for example, [47]). 